Julia Hauck \& Sylvia Linneberg

\title{
Diversität als Selbstverständnis: Die Zukunft der Bibliotheken als Orte des gesellschaftlichen Diskurses
}

\author{
Öffentliche Bibliotheken sind Orte der Begegnung und Plattformen für Aktivitäten der \\ Bürger`innen. Sie stehen für Vielfalt und Inklusion. Durch ihre vielfältige Programm- und \\ Veranstaltungsarbeit wie Lesungen, Workshops oder Diskussionen sind sie essenziell für \\ das kulturelle Leben in vielen Kommunen. (dbv, 2021a, S. 9)
}

Mit diesem Postulat macht das Strategiepapier Öffentliche Bibliothek 2025 des $d b v$ deutlich, wo die Entwicklung der Einrichtungen zukünftig hinführen kann und muss. Diversität sollte kein schmückendes Beiwerk sein, sondern im Selbstverständnis der Bibliotheken verankert. Auch die Rolle der Personalentwicklung in Bibliotheken wird im dbv-Papier (2021a) hervorgehoben: „Eine größere Diversität des Personalkörpers, zum Beispiel in Ausbildung und Herkunft, kann auf den vielfältigen gesellschaftlichen Wandel besser eingehen.“ (S. 12) In diesem Zusammenhang steht auch das althergekommene Berufsbild der Bibliotheksmitarbeiter^innen auf dem Prüfstand. Die diversen Fähigkeiten und Lebensläufe der potentiellen Nachwuchskräfte müssen einbezogen werden. Vielfalt darf nicht nur wertgeschätzt, sondern muss auch gezielt gefördert werden. Dabei ist es wichtig zu verstehen, welche Mechanismen der Ausgrenzung und Diskriminierung dem strukturell entgegenwirken. Bei der Arbeit in der Bibliothek geht es nicht allein darum, die Medien bzw. den Bestand zu hegen und zu pflegen, sondern mit den Besucher*innen in Kontakt zu treten, neue Formen der Partizipation zu ermöglichen und die Arbeit in der Bibliothek als Katalysator gesellschaftlich-demokratischer Teilhabe zu verstehen.

Die Beiträge des Sammelbandes werfen Schlaglichter auf die Gelingensbedingungen Diversitätsorientierter Öffnung und der damit einhergehenden Herausforderungen. Es wird deutlich, dass eine ernst gemeinte Veränderungspolitik die Bereiche Personal, Programm und Publikum umfassen muss. Letztlich sind nicht nur Leitung und Mitarbeiter`innen gefragt, sondern das System Bibliothek als Ganzes muss sich den gesellschaftlichen Entwicklungen stellen. Die Veränderungen betreffen als Querschnittsthema alle Hierarchieebenen.

So aufreibend und anstrengend ein solcher Veränderungsprozess auch sein mag, so gewinnbringend ist das Ergebnis: Bibliotheken bleiben ein Ort für die - 
sich stetig verändernde - (Stadt-)Gesellschaft. Sie reflektieren den Wandel ${ }^{1}$ und bestehen als kommunale Öffentliche Einrichtungen - mit dem Anspruch, den Bürger`innen Teilhabe, Bildung und Kultur zu ermöglichen (Elfering, 2020). Dabei ist es an der Zeit, die Idee einer „interkulturellen Bibliothek“ zugunsten eines intersektionalen Ansatzes von Diversität zu verwerfen - wie nunmehr auch die Umbenennung der entsprechenden Kommission beim dbv bestätigt (dbv, 2021c). Mit der Abwendung von Konzepten wie Interkultur und Integration werden Stereotype über vermeintlich fremde Kulturen aufgebrochen, der Zielgruppen-Begriff grundlegend hinterfragt und neue Perspektiven eröffnet. Menschen sind vielfältig und komplex. Rassismus, Diskriminierung und Benachteiligung sind tief in unseren Strukturen verankert - und keine Einzelfälle (Anti-Diskriminierungsstelle des Bundes, 2017).

\section{Krisen und Konflikte}

Die Veränderungsprozesse hin zur Diversitätssensiblen Öffnung erfordern eine stetige Selbstreflexion und -kritik. Nicht selten fällt es schwer, sich die eigenen Vorurteile einzugestehen und strukturelle Privilegien zu erkennen und $\mathrm{zu}$ hinterfragen. Zumeist sind Öffentliche Bibliotheken heute durch eine relativ homogene Personalstruktur geprägt. Es finden sich „gläserne Decken“ und oftmals wird auf sogenannte diverse Zielgruppen defizitär „herab“ geblickt. Kommen dann Menschen von innen und/oder außen, die grundlegende Strukturen sowie persönliche Identitäten hinterfragen und (neue) Teilhabemöglichkeiten einfordern, entstehen unweigerlich Konflikte.

Oft sehen sich die Mitarbeitenden, die den Prozess innerhalb einer Einrichtung voranbringen wollen, mit deutlichem Widerstand durch die Leitung und/ oder Kolleg^innen konfrontiert. Hier sollte man sich nicht scheuen, Coaching, Supervision oder Mediation in Anspruch zu nehmen. Gerade für die Mitarbeitenden, die Diskriminierung erfahren, haben die Arbeitgeber*innen eine besondere Verantwortung. Ein klares Beschwerdemanagement sollte hier zu kompetenten Ansprechpersonen oder auch Beratungsstellen führen. Präventiv können Empowerment-Trainings und andere sichere Räume eine große Rolle spielen. Die mit Veränderung einhergehenden Konflikte und Hindernisse sind trotz aller negativen Begleiterscheinungen ein gutes Zeichen. Diese Kontroversen und Debatten gehören zur Öffnung dazu, wie Aladin El-Mafaalani (2019) feststellt:

1 Ohnehin bezieht sich das nicht nur auf Veränderungen der stadtgesellschaftlichen Strukturen, sondern auch auf den Medien(nutzungs)wandel oder andere tiefgreifende Veränderungen. 
Solange wir Komplexität und Konflikte negativ konnotieren, spielen alternative Fakten und gefühlte Realitäten eine immer größere Rolle. Vielmehr erfordern die gesteigerte Komplexität der Gesellschaft und das zunehmende Konfliktpotenzial einen Perspektivwechsel. Der Kitt, der die offene Gesellschaft zusammenhält, bildet sich aus Konflikten und dem konstruktiven Umgang mit ihnen. (S. 45)

Um die Entwicklung von Integrationsprozessen und damit auch den Fragen der Identitätspolitik in der Bundesrepublik nachzuspüren, bedient er sich folgender Metaphorik:

Die erste Einwanderergeneration ist noch vergleichsweise bescheiden und fleißig, beansprucht nicht volle Zugehörigkeit und Teilhabe. Sie sitzt überwiegend auf dem Boden beziehungsweise an Katzentischen. Die ersten Nachkommen beginnen sich an den Tisch zu setzen und bemühen sich um einen guten Platz und ein Stück des Kuchens. Nach einer länger andauernden Phase der Integration geht es dann nicht mehr nur um ein Stück des bestehenden Kuchens, sondern auch darum, welcher Kuchen auf den Tisch kommt. (ElMafaalani, 2019, S. 42)

Jetzt ist die Zeit, um zu diskutieren, zu streiten und Kompromisse zu finden. Diskriminierung und Rassismus müssen öffentlich thematisiert und Strategien gefunden werden, um strukturelle Benachteiligung abzubauen. Nur so kann eine demokratische Gesellschaft funktionieren und das Auseinanderdriften von Gruppen in verfeindete Lager aufgehalten werden. Nicht nur in Deutschland herrscht eine zunehmend polarisierte und aggressive gesellschaftliche Atmosphäre, auch in anderen Ländern verschärft sich der Ton. Als literarisches Beispiel sei hier auf das Gedicht The Hill We Climb von Amanda Gorman (2021) verwiesen, durch welches die Lyrikerin die zerrissene US-amerikanische Gesellschaft bei der Inauguration von Joe Biden als Präsident $2021 \mathrm{zu}$ versöhnen suchte. $^{2}$

Neben einem oft aufgeheizten Klima der (nicht-)öffentlichen Debatten um Identitäten, Leitkultur, Gendergerechtigkeit usw., verschärft sich seit Beginn der Corona-Pandemie 2020 die Situation für eine Vielzahl von Menschen (Fratzscher, 2020). Auch für die Öffentlichen Bibliotheken ist der Zugang durch die lang anhaltenden Einschränkungen problematisch (Thiele \& Klagge, 2020). Oftmals werden gerade Besucher*innen ausgeschlossen, die ohnehin mit Barrieren kämpfen: Für wohnungslose Menschen ist kein Aufenthalt möglich, komplizierte Formulierungen in der Kommunikation verhindern den Zugang zu Informa-

2 „We are striving to forge our union with purpose. To compose a country committed to all cultures, colors, characters and conditions of man. And so we lift our gaze, not to what stands between us, but what stands before us. We close the divide because we know to put our future first, we must first put our differences aside. [...] For there is always light, if only we're brave enough to see it. If only we're brave enough to be it.“ (Gorman, 2021) 
tionen für Nicht-Muttersprachler^innen und die digitalen Angebote sowie Kontaktnachverfolgungs-Apps schließen Menschen ohne Zugang zu Technik und das Wissen darum aus.

Auch das Konzept der Bibliothek als Dritter Ort sowie partizipative Formate stehen vor großen Herausforderungen. Wie kann eine Bibliothek als Ort der Begegnung und des Austauschs funktionieren, wenn physische Kontakte nicht möglich sind? So konstatieren Katja Thiele und Britta Klagge (2020, S. 47): „Der in den letzten Jahren viel gepriesene dritte Ort scheint angesichts der CoronaKrise in den Hintergrund zu rücken und die Bibliothek als physischer Ort wird wieder auf das Verleihen von Medien reduziert.“ Viele Einrichtungen sind sich der Problematik alltäglich bewusst und versuchen durch vielfältige Angebote gegenzusteuern (Thiele \& Klagge, 2020; dbv, 2021b). Auch die Stadtgeografin Melike Peterson (2020) sieht die Bibliotheken aufgrund der Pandemie-Einschränkungen in ihren essentiellen Aufgaben bedroht. Gleichzeitig macht die Krise jedoch auch deutlich, warum und wie gerade Öffentliche Bibliotheken zur Grundversorgung der Bürger`innen beitragen:

Und Corona zeigt, wie schützenswert insbesondere Bibliotheken sind. Bibliotheken sind wichtige Orte des Zusammenlebens und der Öffentlichkeit, denn sie erfüllen zentrale Funktionen im Gewebe jeder Stadtgesellschaft: Sie sind Orte der Begegnung zwischen Menschen unterschiedlicher Herkunft und Gesinnung. Sie sind Orte der Bildung und des Lernens, wichtig gerade für Menschen, die sonst keinen Zugang zu einem Computer oder Bildungsmöglichkeiten haben. Und sie sind Orte des Aufenthalts, in denen man Zeit verbringen kann, auch ohne Geld auszugeben. Welche Krisen auch kommen, Bibliotheken helfen uns, sie zu überstehen. (Peterson, 2020)

Auch neue und alte Netzwerke werden vor eine Zerreißprobe gestellt: So sind viele MSO und Initiativen ehrenamtlich tätig, d.h. die Mitarbeit stellt für viele eine zusätzliche Belastung in der Krise dar. Die ehrenamtlichen Kontakte der Bibliotheken leiden unter der Distanz: individuell, aber auch kollektiv durch die erschwerte Kontaktpflege. Die Krise verschärft die Ungleichheit der Gesellschaft (Bundeszentrale für politische Bildung, 2021). Auch die Situation von Migrant ${ }^{\star}$ innen in Deutschland bleibt schwierig (Bendel et al., 2021) ${ }^{3}$. Zudem wurden 2020 fast 80 Prozent mehr Fälle bei der Anti-Diskriminierungsstelle des Bundes registriert als in den Vorjahren (Bundesregierung, 2021) 4 . Es bedarf zukünftig einiger Anstrengungen, um diese negativen Konsequenzen abzufedern.

3 So beschreiben Petra Bendel et al. (2021) drei Szenarien für die Entwicklung bis 2030: 1) „Die Exklusionsgesellschaft: Germans First“, 2) „Die utilitaristische Gesellschaft: Deutschlands neue Gastarbeiter:innen“ und 3) „Die teilhabeorientierte Gesellschaft: Stärker als Viren“ (S. 4 f.)

4 ,2020 zählte die Antidiskriminierungsstelle 1904 Anfragen, die in direktem Bezug zur Pandemie standen. Vor allem rassistische Übergriffe gegen als asiatisch wahrgenommene Men- 
Die langfristigen Folgen der Pandemie für Gesellschaft, Bildung, Kultur und Bibliotheken sind ohnehin auch Ende 2021 noch nicht absehbar.

\section{Verstetigung \& Nachhaltigkeit}

Neben den im Sammelband bereits vorgestellten Maßnahmen gibt es noch zahlreiche andere Möglichkeiten, die Diversitätsorientierte Öffnung in Bibliotheken voranzutreiben. Im Zwischenfazit zum Programm $360^{\circ}$ wird eine Vielzahl von nachhaltigen Maßnahmen für die Diversitätsorientierte Öffnung genannt (vgl. Kulturstiftung des Bundes, 2021):

1. Zielvereinbarungen und Aufnahme von Diversitätskompetenzen in die Anforderungsprofile der Leitungen von Kulturinstitutionen,

2. Etablierung und Verstetigung der Stellen von Diversitätsagent*innen in den Kulturinstitutionen,

3. Sicherung von finanziellen, personellen und zeitlichen Ressourcen,

4. Entwicklung von Ausbildungsprofilen und Qualifizierungsangeboten,

5. Nachwuchsförderung,

6. Etablierung spartenspezifischer Netzwerke,

7. Kooperation mit Akteur*innen und Communitys aus der migrantischen und postmigrantischen Stadtgesellschaft,

8. Einrichtung von Beratungsstellen.

Im Bereich Personal können dies die Erstellung oder Überarbeitung neuer Leitfäden, Wertekodizes und Antidiskriminierungsklauseln oder auch die Gründung einer innerbetrieblichen Beschwerdestelle sein. Für die Verstetigung und Verankerung der Öffnung benötigt es eine Person innerhalb der Einrichtung, die als Diversitätsbeauftragte ${ }^{\star} r$ nach innen und außen fungiert. Die Veränderung der Strukturen kann und darf jedoch nicht an einer Person allein fest gemacht werden und Strukturen müssen personen-ungebunden aufgebaut werden und funktionieren.

Im Bereich Publikum kann die diversitätssensible Neubesetzung von Beiräten, Freundeskreisen usw., eine diversitätssensible Hausordnung oder auch eine professionelle (Nicht-)Besucher*innenforschung den Prozess befördern. Für Öffentliche Bibliotheken sind zudem Leit- und Orientierungssysteme sowie die sogenannten aufsuchenden oder mobilen Angebote essentiell, um Barrieren abzubauen. Die Vernetzung innerhalb der Stadtgesellschaft kann durch die ge-

schen prägten den Beginn der Pandemie. Auch wurde chronisch kranken Menschen und jenen in hohem Alter beispielsweise die Arbeit im Homeoffice verwehrt.“ (Bundesregierung, 2021) 
meinsame Ausformulierung von Kooperationsvereinbarungen auf Augenhöhe unterstützt werden.

Um das Programm zu diversifizieren, ist der Aus- und Aufbau eines fremd-/ mehrsprachigen Bestandes ${ }^{5}$ sowie die Anschaffung vielfaltssensibler Kinderund Jugendmedien notwendig. Hier ist die Einbindung verschiedener Interessensvertretungen für eine Diversitätsorientierte Öffnung wichtig. Weiteres Potenzial bietet die Entwicklung von Angeboten in Gebärdensprache oder Braille sowie für Menschen mit speziellen Einschränkungen, wie z. B. Autismus/Asperger. Bibliotheksarbeit sollte sich nicht nur auf einige Kernzielgruppen konzentrieren, sondern vielfältige Besucher^innen in den Blick nehmen. So spielen Klassismus, Sexismus, antiasiatischer oder antimuslimischer Rassismus, Altersdiskriminierung u.v.m. ebenso eine Rolle, wenn es um die Diversifizierung des Bestandes oder des Veranstaltungsprogramms geht.

Für eine nachhaltige Entwicklung Öffentlicher Bibliotheken sind überregionale Netzwerke von Vorteil, in denen ein Austausch zu Maßnahmen, Herausforderungen und Möglichkeiten stattfinden kann. ${ }^{6}$ Insgesamt muss ein Umdenken stattfinden: Rein quantitative Erfolgskriterien für den Prozess anwenden zu wollen, geht oft an der Realität vorbei, da langfristige Effekte angestrebt werden.

Diversität ernsthaft $\mathrm{zu}$ fördern und $\mathrm{zu}$ fordern, bedarf einer Kraftanstrengung aller Beteiligten, um die Veränderungen der (Stadt-)Gesellschaft aufzugreifen und das Zusammenleben, -lernen und -wachsen positiv zu gestalten. Öffentliche Bibliotheken müssen sich von innen heraus wandeln. Sie sind Plattformen, um für eine gerechtere Informations- und Wissensgesellschaft einzustehen. Sie können ein Ort sein, an dem ein ${ }^{\star} e$ jede ${ }^{\star} \mathrm{r}$ „sein“ kann; ein Raum, um sich einzubringen und den Horizont zu erweitern. Diversitätsentwicklung ist eine einmalige Chance für die Zukunft der Bibliotheken. „Bibliotheken sind ein wichtiger Motor für Chancengerechtigkeit in einer vielfältigen Gesellschaft. Keine andere Einrichtung erreicht mit ihren niederschwelligen Angeboten so viele unterschiedliche Zielgruppen wie Bibliotheken“, lautet es in der Pressemitteilung des dbv zur Gründung der Kommission „Bibliotheken und Diversität“ (dbv, 2021c). Die zentralen Fragen und damit verbundenen Aufgaben für Öffentliche Bibliotheken sind: Wie wollen wir in Zukunft zusammenleben? Wie kann auf eine gerechte Teilhabe ohne Barrieren und Diskriminierung hingearbeitet werden? Und vor allem: Was können und müssen Öffentliche Bibliotheken dafür leisten?

5 Auch die Verlage müssen sich bewegen und Bibliotheken können hier ihren Einfluss nutzen. 6 Mittlerweile finden sich einige Initiativen in den Bibliotheksnetzwerken, wie Veranstaltungen der AG Barrierefreiheit des dbv. 
Der vorliegende Sammelband zeigt beispielhaft, welche ersten Schritte zur Veränderung notwendig sind. Wie zukünftig eine Diversitätssensible Bibliothek gestaltet werden kann, ist die zentrale Frage für das Weiterbestehen der Bibliotheken als Öffentliche Einrichtungen der (Stadt-)Gesellschaft. In diesem Sinne muss auch die kulturpolitische Ausrichtung der Kommunen und Institutionen neu verhandelt werden. Mancherorts finden sich dafür bereits erfolgsversprechende Initiativen, wie bspw. in Hamburg, wo sich derzeit ein Netzwerk für Diversität in Kultur und Kultureller Bildung formiert (www.nediku.de). ${ }^{7}$

Letztendlich bleibt die Diversitätsorientierte Öffnung ein Prozess, dessen Maßnahmen und Ziele immer wieder hinterfragt, neu justiert und ausgebaut werden müssen. Ob im kleinen Team oder auf höchster Hierarchieebene: Es sind alle gefragt. Packen wir es an!

\section{Literaturverweise}

Antidiskriminierungsstelle des Bundes und der in ihrem Zuständigkeitsbereich betroffenen Beauftragten der Bundesregierung und des Deutschen Bundestages. (2017). Diskriminierung in Deutschland - Dritter Gemeinsamer Bericht der Antidiskriminierungsstelle des Bundes und der in ihrem Zuständigkeitsbereich betroffenen Beauftragten der Bundesregierung und des Deutschen Bundestages. http://www.antidiskriminierungsstelle.de/Shared Docs/Downloads/DE/publikationen/BT_Bericht/Gemeinsamer_Bericht_dritter_2017.pdf (1.4.2021).

Bendel, P., Bekyol, Y. und Marlene Leisenheimer. (2021). Auswirkungen und Szenarien für Migration und Integration während und nach der COVID-19 Pandemie. MFI Erlangen. https://www.covid-integration.fau.de/files/2021/04/studie_covid19-integration_fau.pdf (1.4.2021).

Bundesregierung. (2021, November 5). Jahresbericht der Antidiskriminierungsstelle. https:// www.bundesregierung.de/breg-de/suche/mehr-aufmerksamkeit-gegen-diskrimi nierung-1913048 (1.4.2021).

Bundeszentrale für politische Bildung. (2021). Datenreport 2021. Ein Sozialbericht für die Bundesrepublik Deutschland. Statistisches Bundesamt (Destatis), Wissenschaftszentrum Berlin für Sozialforschung (WZB), Bundesinstitut für Bevölkerungsforschung (BiB). https://www.wzb.eu/system/files/docs/sv/k/dr2021_0.pdf (1.4.2021).

7 Akteur^innen aus Vereinen, Verbänden, Zusammenschlüssen und Institutionen wie auch Einzelpersonen aus der Stadtteilkultur, den darstellenden Künsten, der freien Theaterszene, der Kinder- und Jugendkultur und des Globalen Lernens haben sich darin zusammengeschlossen. Das „Netzwerk der Netzwerke“ fordert u. a. die öffentliche Finanzierung von Prozessbegleitungs- und Qualifizierungsmaßnahmen, um in der gesamten Hamburger Kulturszene Strategien zur diversitätsorientierten Öffnung auf den Weg zu bringen. 
dbv. (2021a). Öffentliche Bibliothek 2025: Leitlinien für die Entwicklung der Öffentlichen Bibliotheken. https://www.bibliotheksverband.de/fileadmin/user_upload/DBV/publikatio nen/Positionspapier_\%C3\%96B_2025_FINAL_WEB.pdf (1.4.2021).

dbv. (2021b). Spotlight Corona. Bibliotheksportal. https://bibliotheksportal.de/spotlightcorona (1.4.2021).

dbv. (2021c, Mai 18). Diversity-Tag 2021: Bibliotheken, Orte der kulturellen Vielfalt und Toleranz [Pressemitteilung]. https://www.bibliotheksverband.de/dbv/presse/presse-de tails/archive/2021/may/article/bibliotheken-orte-der-kulturellen-vielfalt-und-toleranz. html?tx_ttnews[day]=18\&cHash=c538ad574fd56b9aa1698745e1c16cd4 (1.4.2021).

Elfering, M. (17.5.2021). Bibliotheken und Bildungschancen - Wie Zugang zu Wissen das Leben verändert. In Zeitfragen. Deutschlandfunk Kultur. https://www.deutschlandfunkkultur. de/bibliotheken-und-bildungschancen-wie-zugang-zu-wissen-das.976.de.html?dram:arti cle_id=497338 (1.4.2021).

El-Mafaalani, A. (2019). Alle an einem Tisch. Zwischen Teilhabe und Diskriminierung. In Politik und Zeitgeschichte, 9-11(Identitätspolitik), S. 41-45.

Fratzscher, M. (2020, Dezember). Nicht das Virus ist ungerecht. Zeit Online. https://www.zeit. de/wirtschaft/2020-12/soziale-ungerechtigkeit-pandemie-coronavirus-arbeit-gesund heitssytem-hilfsprogramm (1.4.2021).

Gorman, A. W. (2021). The Hill We Climb: An Inaugural Poem for the Country.

Kulturstiftung des Bundes. (2021). Diversität als Zukunftsfaktor. Empfehlungen für eine nachhaltige Diversitätsentwicklung in Kulturinstitutionen aus dem Programm $360^{\circ}$ - Fonds für Kulturen der neuen Stadtgesellschaft. https://www.kulturstiftung-des-bundes.de/filead $\mathrm{min} /$ user_upload/download/download/360/210511_KSB_360Grad_Positionspapier_A4_ finale_Version.pdf (1.4.2021).

Petersen, M. (2020, September 23). Bibliotheken und Corona - Zerbrechliche Orte des öffentlichen Raums. In Politischer Feuilleton. Deutschlandfunk Kultur. https://www.deutschland funkkultur.de/bibliotheken-und-corona-zerbrechliche-orte-des.1005.de.html?dram:arti cle_id=484533 (1.4.2021).

Thiele, K., \& Klagge, B. (2020). Öffentliche Bibliotheken als dritte Orte und Bildungsgerechtigkeit in Zeiten von Covid-19. Bibliothek Forschung und Praxis, 44(3), 552-559. https://doi. org/10.1515/bfp-2020-2023 (1.4.2021). 